\title{
Bihemispheric Paradoxical Cerebral Embolism in a Patient with Pulmonary Thromboembolism and Presumptive Fistula Right-to-Left Shunt
}

\author{
Silméia Garcia Zanati Bazan, MD, PhD,* Gabriel Pereira Braga, MD, PhD, $†$ \\ Gustavo José Luvizutto, MD, MSc, † André Petean Trindade, MD, PhD, $\ddagger$ \\ Octávio Marques Pontes-Neto, MD, PhD, $§$ and Rodrigo Bazan, MD, PhD†
}

\begin{abstract}
We report a case of a bihemispheric paradoxical cerebral embolism in a patient with pulmonary thromboembolism and presumptive pulmonary arteriovenous fistula. The echocardiogram showed no intracardiac shunt, and the transcranial Doppler (TCD) revealed spontaneous microembolic signals in the middle cerebral arteries (MCAs), and late passage of a higher number of microembolic signals in the MCAs, compatible with right-to-left shunt (RLS). The TCD and the echocardiogram were useful for identifying the RLS when rapid neurological deterioration occurred. Key Words: Stroke - paradoxical embolism—echocardiogram—-transcranial Doppler. (c) 2016 National Stroke Association. Published by Elsevier Inc. All rights reserved.
\end{abstract}

\section{Introduction}

Paradoxical embolisms due to cardiac or pulmonary shunting are an important but rare cause of stroke in younger patients, with an estimated rate of $1 \%$ in patients with a cryptogenic stroke or transient ischemic attack (TIA). The most common cause of a paradoxical embolism is an intracardiac right-to-left shunt (RLS) through

From the *Department of Internal Medicine, Botucatu Medical SchoolUNESP, São Paulo State University, São Paulo, Brazil; +Department of Neurology, Botucatu Medical School-UNESP, São Paulo State University, São Paulo, Brazil; łDepartment of Tropical Diseases and Diagnostic Imaging, Botucatu Medical School-UNESP, São Paulo State University, São Paulo, Brazil; and §Department of Neuroscience and Behavior, Ribeirão Preto Medical School-USP, São Paulo State University, São Paulo, Brazil.

Received February 3, 2016; revision received March 14, 2016; accepted March 19, 2016.

Address correspondence to Gustavo José Luvizutto, MD, MSc, Departamento de Neurologia, Psicologia e Psiquiatria, Faculdade de Medicina de Botucatu-UNESP, Distrito de Rubião Júnior s/n, 18618-970

Botucatu, São Paulo, Brazil. E-mail: gluvizutto@fmb.unesp.br.

1052-3057/\$ - see front matter

(C) 2016 National Stroke Association. Published by Elsevier Inc. All rights reserved.

http://dx.doi.org/10.1016/j.jstrokecerebrovasdis.2016.03.038 a patent foramen ovale (PFO), but a paradoxical embolism can also be caused by an extracardiac RLS through a pulmonary arteriovenous fistula (PAVF). ${ }^{1,2}$

Transthoracic and transesophageal echocardiograms are performed to identify the source of embolic strokes and they are usually focused on identifying an intracardiac shunt source, such as a PFO. Transcranial Doppler (TCD) monitoring can be done at the bedside to investigate the cause of a cryptogenic stroke. TCD is a unique method that evaluates the embolus sources in real time, and it offers an auxiliary method for the diagnosis of possible embolic sources. ${ }^{3}$

Intrapulmonary shunts are a less common cause of cerebral embolism; they are a less recognized cause of paradoxical emboli; and their impact on the risk of stroke and TIA is still incompletely understood. ${ }^{4,5}$ Herein, we present a case of a bihemispheric paradoxical cerebral embolism in an older patient with a pulmonary thromboembolism caused by a presumptive PAVF.

\section{Case Report}

A 66-year-old male presented to the hospital with a sudden onset of loss of consciousness, central cyanosis, 


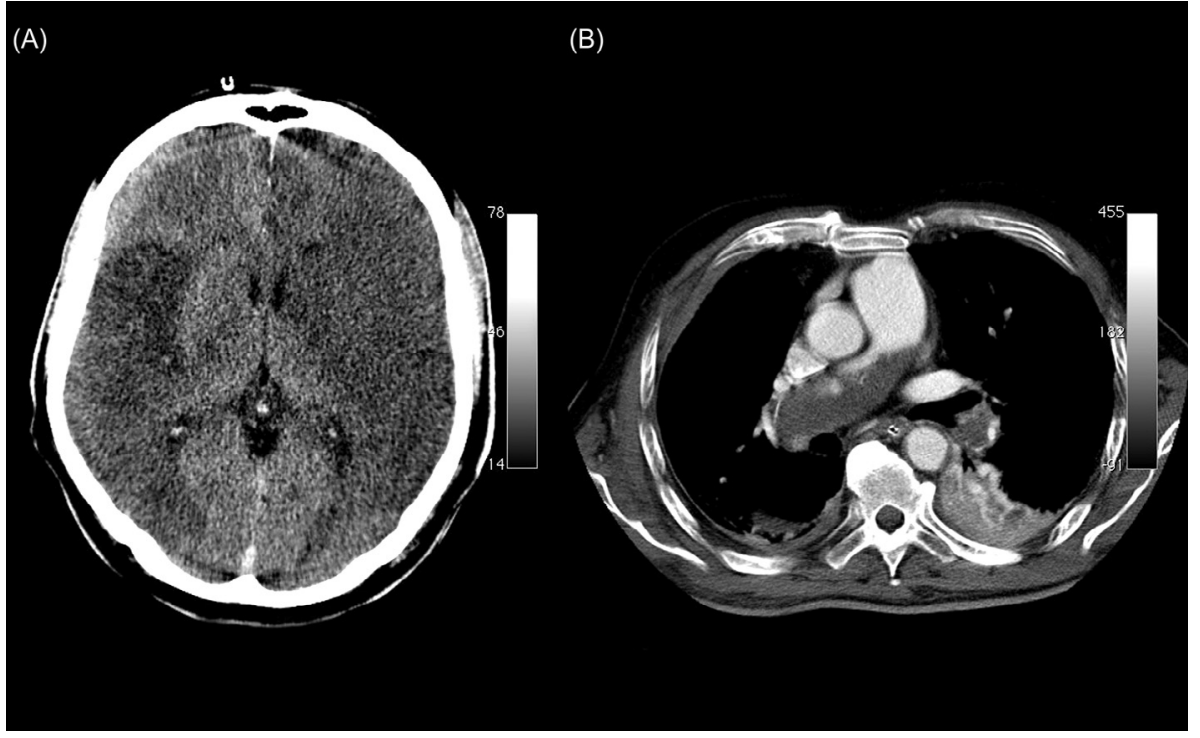

Figure 1. (A) CT scan of the brain shows massive bilateral ischemic stroke. (B) CT angiography of the chest shows massive pulmonary thromboembolism. Abbreviation: $C T$, computed tomography.

mydriasis, and decerebrate posturing. He had a history of a pulmonary thromboembolism 10 years prior. There is no family history reported. The patient was stabilized and placed on mechanical ventilation. The electrocardiogram showed right atrial overload without identified arrhythmias. Computed tomography (CT) scan of the brain (Fig 1, A) demonstrated a massive bilateral ischemic stroke and CT angiography of the chest (Fig 1,
B) revealed a massive pulmonary thromboembolism. A TCD study was performed in the emergency room and showed the presence of spontaneous microembolic signals (MES) in the middle cerebral arteries (MCAs). Next, simultaneous transthoracic echocardiography and TCD monitoring that included the injection of agitated saline (microbubble test) were performed (Fig 2, A). The echocardiogram (Fig 2, B) showed right ventricular

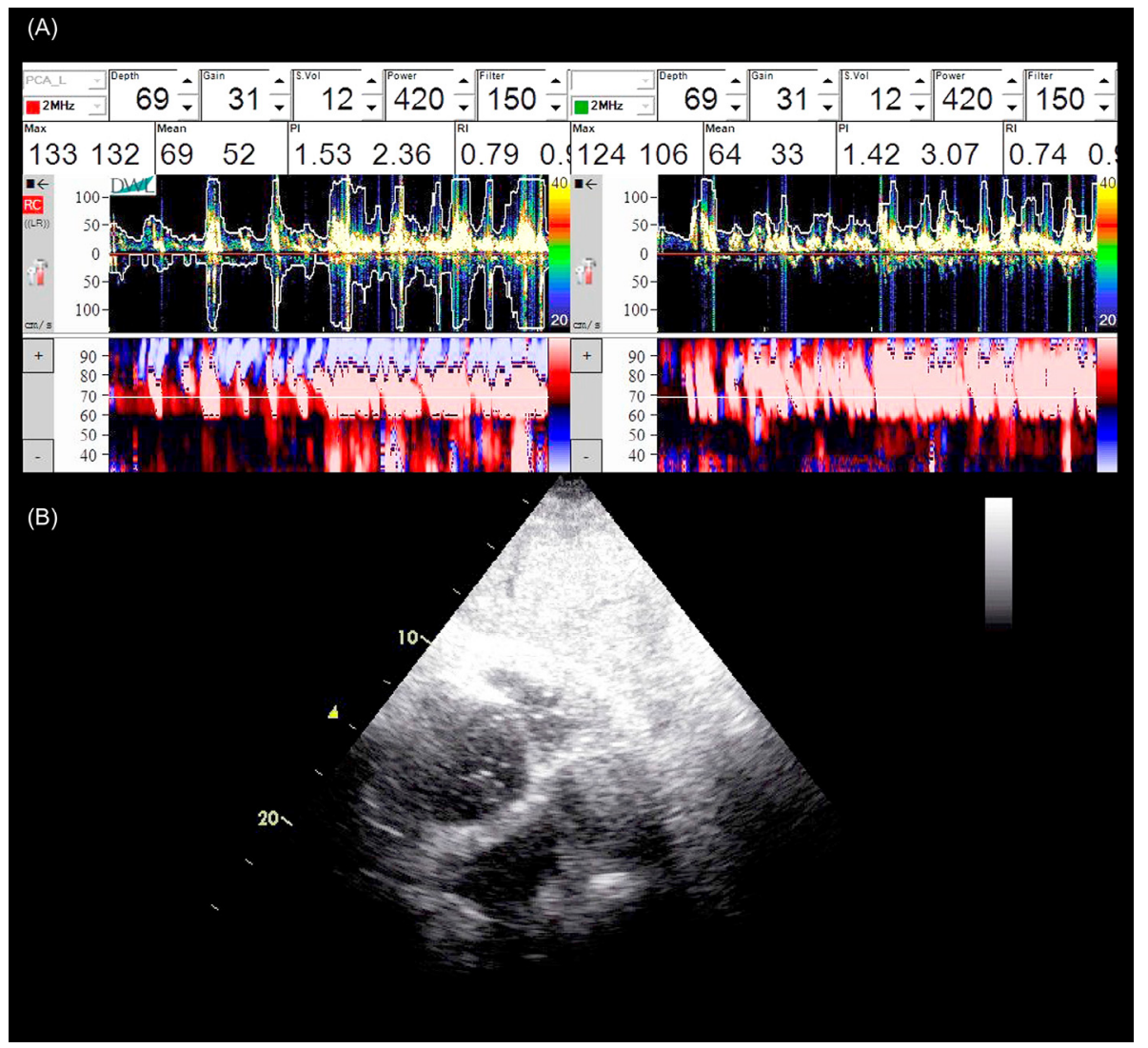

Figure 2. (A) Transcranial Doppler shows the presence of spontaneous high-intensity transient signs in the middle cerebral arteries after bubble test. (B) Echocardiogram shows no passage through a patent foramen ovale and pulmonary hypertension. 
dysfunction and increased pressure in the right heart chamber, denoting a pattern of pulmonary hypertension. No evidence of passage through a PFO was observed. The TCD exam showed late passage ( $>18$ seconds) of a higher number of MES (>10) in both MCAs, compatible with a significant RLS. Invasive pulmonary angiography was not performed because the patient was hemodynamically unstable; he suffered a fast neurological deterioration and died within 48 hours of admission.

\section{Discussion}

The patient's CT scan was performed during the acute phase of the stroke and showed a massive bilateral ischemic stroke of the anterior circulation. This type of presentation during the acute phase is unusual; it occurs in approximately $4 \%$ of all ischemic strokes; and it can suggest an embolic mechanism. ${ }^{6}$ PAVFs are rare pulmonary vascular malformations that result in direct communication between the branches of the pulmonary artery and veins; they have an incidence of 2-3 per 100,000 in the population. ${ }^{7}$ PAVFs do not affect cardiac hemodynamics and most patients are asymptomatic; however, if the RLS is large, this malformation can cause desaturation, dyspnea, cyanosis, clubbing, and polycythemia. The associated central nervous system complications of PAVFs can include migraines, TIAs, abscesses, seizures, and ischemic strokes. ${ }^{8,9}$

The etiology of a RLS is only well defined when the presence of the "in transit" embolus is documented. During the acute evaluation of a stroke patient, diagnostic methods such as TCD and transthoracic echocardiography can be combined to screen for RLS and to differentiate between intracardiac and extracardiac sources. ${ }^{3}$ In this reported case, the transthoracic echocardiogram showed increased pressure in the right heart chamber and excluded a PFO.

The TCD monitoring of this patient detected expressive MES in both MCAs, including the later passage in the brain's arterial circulation. Some investigators have demonstrated that TCD can aid in the diagnosis of embolic sources; both the presence and the timing of MES help determine the etiology. The timing of the MES can increase the specificity of the diagnosis; a microembolic passage time of about 15 seconds can predict PAVFs. ${ }^{4}$ The MES findings in this patient were present bilaterally and in large numbers with a late time of passage; all of these findings suggest an extracardiac source as the cause of stroke and the presumptive diagnosis of PAVF.

PAVFs can be an independent predictor of extracardiac sources of ischemic stroke, with variable rates (18\%-60\%).
The "gold standard" for the diagnosis of PAVF is pulmonary angiography, but a chest radiography can be used as a screening tool and will reveal abnormalities in about $45 \%-98 \%$ of patients with PAVF. ${ }^{10}$ In this case, the CT angiography of the chest was the screening tool of choice because it could be performed quickly in this hemodynamically unstable patient who suffered a rapid neurological deterioration.

\section{Conclusion}

The combination of a probable fistula and pulmonary thromboembolism is an uncommon cause for an extensive bilateral ischemic stroke and has not been reported in the literature often. The TCD and the transthoracic echocardiography with saline contrast were useful tools for identifying the presence of an RLS in this case of an unstable patient who experienced rapid neurological deterioration.

\section{References}

1. Loscalzo J. Paradoxical embolism: clinical presentation, diagnostic strategies, and therapeutic options. Am Heart J 1986;112:141-145.

2. Reguera JM, Colmenero JD, Guerrero M, et al. Paradoxical cerebral embolism secondary to pulmonary arteriovenous fistula (letter). Stroke 1990;21:504-505.

3. Kimura K, Minematsu K, Wada K, et al. Transcranial Doppler of a paradoxical brain embolism associated with a pulmonary arteriovenous fistula. AJNR Am J Neuroradiol 1999;20:1881-1884.

4. Horner S, Niederkorn K, Gattringer T, et al. Management of right-to-left shunt in cryptogenic cerebrovascular disease: results from the observational Austrian paradoxical cerebral embolism trial (TACET) registry. J Neurol 2013;260:260-267.

5. Wozniak L, Mielczarek M, Sabiniewicz R. Paradoxical brain embolism in a young man: is it only patent foramen ovale? Neurol Neurochir Pol 2015;49:61-64.

6. Saito K, Hiroshi Moriwaki H, Oe H, et al. Mechanisms of bihemispheric brain infarctions in the anterior circulation on diffusion-weighted images. AJNR Am J Neuroradiol 2005;26:809-814.

7. Hodgson $\mathrm{CH}$, Keye RL. Pulmonary arteriovenous fistula and hereditary telangiectasia. Dis Chest 1963;43:449-455.

8. Majeed FA, Ghumman AQ, Chatha SS. Pulmonary arteriovenous malformation (PAVM): rare cause of hemoptysis. J Ayub Med Coll Abbottabad 2013;25:100-102.

9. Cottin V, Chinet T, Lavole A, et al. Pulmonary arteriovenous malformations in hereditary hemorrhagic telangiectasia: a series of 126 patients. Medicine (Baltimore) 2007;86:1-17.

10. Moussouttas M, Fayad P, Rosenblatt M, et al. Pulmonary arteriovenous malformations: cerebral ischemia and neurologic manifestations. Neurology 2000;55:959-964. 